\title{
The role of patient-led education initiatives in medical education
}

\author{
This article was published in the following Dove Press journal: \\ Innovation and Entrepreneurship in Health \\ II June 2015 \\ Number of times this article has been viewed
}

\section{Ceridwen Coulby Vikram Jha}

School of Medicine, University of Liverpool, Liverpool, UK
Correspondence: Vikram Jha Undergraduate School of Medicine, University of Liverpool, 218 Cedar House, Ashton Street, Liverpool, Merseyside L69 3GE, UK

Email vikramjha@liverpool.ac.uk
Abstract: There are health care policy, service delivery, and educational drivers for the involvement of patients in medical education. In this article, we explore some of these drivers and the current theoretical paradigms through which patient involvement in medical education is examined. The roles that patients currently play in medical education are considered, along with aims, benefits, and potential drawbacks of involvement for patients, students, and medical educators. A review of current literature and the limitations thereof is included. The gap between policy and practice is considered, and potential reasons for this gap are suggested. Lastly, the impact of patient-led education is considered, and how patient-led education can best be used to transform student learning.

Keywords: patient involvement, medical education, patient-led education, patients as teachers

\section{Introduction}

There is a drive from the General Medical Council to involve patients more explicitly in medical education. This has resulted in the development of a range of innovative interventions utilizing the expertise of patients. A number of terms have been employed to describe patient involvement in education, including "real" patients, "simulated" patients, "standardized", patients and "patient substitutes" (eg, mannequins, videos). While many of these terms overlap, they are distinct, each with a different definition and purpose.

Simulated patients are generally laypeople trained to portray patients with specific health problems realistically. ${ }^{1}$ Simulated patients are widely employed in communication, consultation, and examination skills training for undergraduate medical students. ${ }^{2}$ The purpose of this is to provide students with a safe environment in which to practice and improve their clinical and interpersonal skills through role play and feedback prior to their work-based experiences with "real" patients. This reduces risk to both patients and students, and scenarios can be tailored to student learning needs. ${ }^{3}$

Simulated patients are also widely employed for formative and summative assessment purposes, in which they can also be referred to as "standardized patients". Standardized patients are required to portray a specific patient with a medical condition for assessment purposes, most commonly formative and summative Objective Structured Clinical Examination (OSCE). The simulated patient is provided with a detailed history and symptoms, and must present these in a standardized way to multiple students to ensure fairness and consistency in examinations. ${ }^{4}$ Specific training is provided to these simulated or standardized patients to improve the reliability of exams. ${ }^{5}$ There is also 
evidence that when simulated patients' judgments of student performance are included in the OSCE-marking scheme, the exam itself can be more reliable. ${ }^{6}$

The term "patient substitutes" can be used to describe any intervention that does not involve interaction with "real" patients; as such, even simulated patients can be referred to in this way. ${ }^{7}$ However, more usually this term is used to describe task trainers that can be used by students to practice practical skills, such as genital examination or highfidelity simulation environments that utilize "intelligent" mannequins to simulate emergency situations and trauma. ${ }^{3}$ Another patient substitute that has become increasingly popular is the use of the "virtual patient". ${ }^{8}$ Virtual patients are computer-based and vary in their level of interaction, from preprogrammed patient stories used to develop student clinical reasoning to projected images of a patient with voice-recognition technology to support students practice their history taking. ${ }^{9}$

However, none of these definitions can be described as patient-led. It is the involvement of real patients, portraying their own experience of health care either personally or indirectly through their relatives, that is of particular interest to us when discussing patient-led interventions.

Traditional medical training has focused on providing instruction using the perspective of a "doctor lens" with less emphasis on what it is like to live with a condition or experience health services from a "patient lens". Patients have therefore been used as passive props to facilitate training. More recently, greater emphasis has been given to a more "active" form of patient involvement. ${ }^{10,11}$

\section{Drivers for patient-led education}

There are a number of drivers for greater patient involvement in medical education. These are intrinsically linked to health care policy, health service delivery, and patient-led medical education literature.

\section{Health care policy}

The Department of Health document Equity and Excellence: Liberating the $N H S^{12}$ emphasized the importance of bringing the patient back into the center of all health care: "Nothing about me without me". This document included the need for patients to be involved in health care policy and service delivery.

In addition, the national rollout of the Expert Patients Programme brought to our attention the need to involve patients with chronic conditions in educating students and trainees. This was due to the recognition that some of these patients who have a lived experience of these conditions know more about the condition, and in particular living with the condition than medical practitioners. The recent high-profile cases involving mismanagement of patients in National Health Service (NHS) trusts (Mid Staffordshire) have further highlighted the importance of patient involvement.

According to NHS England:

Patients, the public and staff should be engaged throughout the development of proposals from their very early initiation through to implementation. Engagement should seek to build an on-going dialogue with the public, where they have an opportunity to shape and contribute to proposals $[\ldots] .{ }^{13}$

With this in mind, the traditional use of patients as passive props in medical education must be expanded to reflect these values.

\section{Health care delivery}

In an article referring to "the four horsemen of the medical education apocalypse", Albanese et $\mathrm{al}^{14}$ highlighted four major consequences for medical education due to the changes in health services delivery across Western societies. These were: teaching patient shortages, teacher shortages, conflicting systems, and financial problems.

If we look at these in the context of how the NHS and other health services are currently organized, the similarities are obvious. There is a real issue with medical students getting opportunities to see a wide variety of medical conditions and all their complex presentations during hospital placements. Patients with long-term conditions are increasingly treated in the community. In addition, patients present with complex comorbidities rather than isolated conditions, and this makes illness scripts difficult to create within learners' cognitive development. There is a national debate on workforce planning of the future. With the new consultant contract in the UK, consultants no longer have the liberty to take time to teach students and juniors. In the current financial climate, there is an ever-present conflict between running the health service as a business and identifying time for students/trainees to receive training.

In light of this complex set of circumstances, it is clear that patient-led education initiatives can have a real impact. By sourcing more patients as teachers in the classroom, hospital, community, and in their own homes, the shortage of patient teachers can be reduced, along with the impact of teacher shortages, conflicting health care-delivery systems, and financial problems. 


\section{Patient-led medical education literature}

Patient involvement in education is a growing field of activity within medical education as a result of these health care drivers, and this is reflected in the literature. In our review of patient-led education literature, we searched such databases as Medline, the Educational Resources Information Center (ERIC), Embase, Topics in Medical Education (TIMELit), PsycInfo, and the Cumulative Index to Nursing and Allied Health Literature (CINAHL) using the terms "patient\$", "educat\$", "medical education", "involv\$”, "teach\$", "learn\$”, "service user", “carer\$”, "student”, "undergraduate", "medical", "postgraduate", "curriculum", and "assess\$". We then reviewed the abstracts and selected papers that were relevant to "real patient"-led education initiatives.

\section{Theoretical paradigm for studying patient involvement in education}

While the majority of the literature in the patient-involvement field is not theoretically based, there are some notable exceptions. These include Lave and Wenger's theory of situated learning, ${ }^{15}$ in particular the concept of legitimate peripheral participation and communities of practice, and Goffman's dramaturgy. ${ }^{16}$ Both of these models have been used by Rees et $\mathrm{a}^{17,18}$ and Monrouxe et $\mathrm{al}^{19}$ in respective studies.

In Rees et al's 2007 paper, ${ }^{17}$ Lave and Wenger's concept of legitimate peripheral participation is used to explore the roles students and patients are afforded by the clinical communities of practice. Legitimate peripheral participation is the term Lave and Wenger used to describe how learners are slowly integrated into the community they are training to become a part of. The learner starts their learning journey from the point of legitimate peripheral participation. They are allowed to be in the environment, but they do not yet fully belong, as they have yet to become full members (or participants) of that community of practice. They need to learn the language, identity, roles, and skills of those who already belong. ${ }^{15}$ Rees et al identified that when students learn from patients in the clinical setting, they can feel challenged by the patients' empowerment and their own disempowerment. When this occurs, students can feel that their sense of identity and "belonging" to the clinical community of practice is compromised. Interestingly, Rees et al also noted that students are more comfortable when patients are "brought in"17 to the university for teaching. This can be explained by identifying that in a university setting, students are full participants in the student community of practice, and the patient becomes the outsider, temporarily granted legitimate peripheral participation.
In reality, both students and patients experience varying degrees of participation within the clinical community of practice, dependent on the situation and individuals involved. When patients' expertise is recognized by clinicians for those living with and managing chronic conditions, the patient has a greater degree of belonging than the student. However, generally students see themselves (and are seen as) the middle point of the hierarchy. Rees et al identified that this position is sometimes regarded as that of a "broker" ${ }^{17}$ between the patient and clinical worlds.

The use of this theoretical lens allows us to explore possible explanations for the difficulty of translating patientcenteredness and involvement from theory into practice. Raising awareness among educators and learners of the struggle students experience when developing their professional identity and moving toward full participation could help to build more supportive learning environments, while recognizing patients as legitimate participants within the clinical community of practice, even in a peripheral way, would improve the level of patient involvement in medical education and promote more patient-centered care.

In another study conducted by Rees et al examining metaphorical analysis of talk about student/doctor-patient relationships, ${ }^{18}$ they discovered that the language used by students, patients, and doctors when discussing their relationships was laden with hierarchical and war metaphors, demonstrating that patient involvement and patient-centeredness as the norm is still culturally a long way off.

Monrouxe et $\mathrm{al}^{19}$ examined patient involvement in bedside teaching through Goffman's dramaturgy theory. In this theory, interactions are viewed in terms of theater, with individuals playing the roles of director, performer, audience, nonperson, and prop. Goffman also considers the setting of the interaction and the use of back stage and front stage among performers and directors. The role of secrets is also explored, ie, information that is known to some of those involved but not all. ${ }^{16}$

Monrouxe et al found that even within one encounter, individuals involved can play multiple roles. For example, the doctor can potentially alternate between the role of director, performer, and audience, while the patient may play the role of director, performer, audience, nonperson, and prop, dependent on the situation and the attitudes of the individuals involved. Students often found themselves in the role of performer or audience. Within all encounters, secrets were present as the doctor and student discussed the encounter in medical terms. These discussions often took place as backstage activities, with the student and doctor discussing the 
patient's symptoms, etc, in low voices while simultaneously engaging in front-stage conversation with the patient. The effect of this backstage talk and secrets can leave the patient feeling excluded from their own care, a nonperson, or prop in the student's learning experience. Monrouxe et al suggested the insights generated by this study could be used to shape encounters between doctor, student, and patient to encourage more patient-centered care, but also as a learning tool to gain greater understanding of the student's medical knowledge. They suggested that "students could explain their actions and findings to both patients and doctors using medical language plus a lay translation". ${ }^{19}$

What is clear from both of these studies is the role that the application of theory to patient-involvement initiatives can help us to understand why the theory/practice gap of patient involvement exists and provide insights into how we might address these issues.

\section{Patient roles in medical education}

Traditionally, patients have mainly served as objects in bedside teaching. ${ }^{17}$ However, in their more active role within medical education, the most common role patients fulfill is that of teachers. ${ }^{20}$

\section{Patients as teachers}

Patients have been involved in teaching clinical examination skills since the $1960 \mathrm{~s},{ }^{21}$ initially as "programmed patients" in simulated neurology consultations (what we would refer to today as simulated patients), and then latterly as themselves where mothers were able to provide their own children's clinical histories to students, providing students with opportunities to practice their pediatric interviewing skills. ${ }^{22}$

Patients' expertise has since been widely employed to deliver clinical skills teaching and assessment in such areas as gynecology and musculoskeletal examination. ${ }^{10}$ In this capacity, patients have mainly contributed to the skill development of students.

A more recent teaching role for patients is their involvement with communication and consultation skills teaching. ${ }^{23,24}$ Within these roles, patients are not just contributing to practical skill development but also to the development of student attitudes toward patients in a personal and professional capacity.

Lastly, the use of patient narratives to provide students with a "patient perspective" on medical conditions and the biopsychosocial implications of living with disease has grown popular, and there is evidence that students and patients find this a powerful learning experience, ${ }^{25}$ either in the classroom, community, or the patient's own home. Patient narratives enable patient teachers to contribute to all educational domains, knowledge, skills, and attitudes, demonstrating greater impact in terms of meaningful, memorable learning experiences $^{26,27}$ and level of patient involvement. ${ }^{28}$

\section{Evidence base for patient as teachers}

A systematic review of patient involvement in teaching showed effectiveness in terms of increased learner satisfaction and improved communication skills among health care professionals. ${ }^{11}$

The increase in learner satisfaction is explained by students in a variety of ways, such as enhancement of perspective, context, motivation, and confidence, ${ }^{26}$ along with change in attitudes, understanding, and assumptions toward the needs of those living with disease. ${ }^{10}$

Recent papers have demonstrated no notable difference between the educational attainment of students taught clinical examination of the musculoskeletal system by patients and those taught by clinicians. ${ }^{29}$ This is mirrored in the attainment of trainees taught patient safety by clinicians and those taught by patients. ${ }^{27}$ However while the educational outcome was achieved equally well through either method, student and trainee satisfaction was lower for those taught by patients.

It would seem that while students value patient-led teaching when the objective is to understand the patient perspective, they do not value patient-led teaching as much as clinician-led teaching when it comes to skill or knowledge acquisition. This attitude is reflective of the importance of power, status, identity formation, and paternalistic attitudes still at work in medicine. ${ }^{17-19,30}$

There are concerns that while willing to lend power to patients for specific purposes, the medical profession is not willing to relinquish its own position as the ultimate authority. Patients as educators place students in the role of learner rather than carer, which can be threatening to their sense of identity. ${ }^{31,32}$ The cooperation-guidance model ${ }^{33}$ (where the doctor tells the patient what must be done and the patient is expected to comply) between patients and doctors is so embedded in the identity and culture of doctoring that making the leap to receiving guidance from patients is a huge paradigm shift. Medical educators can experience similar feelings. ${ }^{17}$ This uneasy situation perpetuates the perception among many that within health care, often only "lip service" is paid to patient involvement. ${ }^{1}$

While the majority of the published literature is concerned with the benefits of patient-led education to students, there are also benefits to patients fulfilling teaching roles. These include 
companionship, improved self-esteem, empowerment, and the altruistic aim of improving future patient experiences and helping the professionals of the future. ${ }^{10}$ Additional benefits, such as developing a better knowledge of their own health problems, developing a comprehensive illness narrative, better health outcomes, and deeper understanding of health services and doctor-patient relationships, have also been reported. ${ }^{11}$

Few patients have reported negative consequences of being involved in medical education. However, examples of patients feeling embarrassed or exposed, ${ }^{34}$ feeling intimidated by being outnumbered by students and teachers, ${ }^{17}$ and feeling exploited and objectified ${ }^{35,36}$ have been reported.

Both students and patients can feel insecure in these patient-led training situations. Students can worry about being a burden to patients, ${ }^{37}$ or believe that they are exploiting the patient for their own learning purposes. ${ }^{17}$ Occasionally, students feel that patients take advantage of their student state to try and get more information or influence doctors on their behalf. ${ }^{17}$ In turn, patients can be concerned about students judging them, portraying them inaccurately in assignments and about their confidentiality and consent. ${ }^{20}$ They can also become upset if students do not seem interested in them, ${ }^{27}$ which can be particularly distressing if sharing a personal illness narrative in a classroom setting, or feel pressured into accommodating students for bedside teaching when they do not feel up to it. ${ }^{34}$

While the literature demonstrates that patients can be involved in medical education as teachers, what is less clear is what the real purpose of such an involvement is.

In Jha et al's systematic review (2009) of the "strategies and interventions for the involvement of real patients in medical education", ${ }^{11}$ the aim of patient-involvement studies was examined. Examples of the rationale for patients as teachers included previous literature demonstrating equivalence between patient and clinician-led teaching of examination (the implication being on limitations of faculty teaching time and cost associated with that), that students and patients enjoyed and benefited from it, and that patients brought a perspective of living with disease that clinicians could not.

As we can see, several different purposes are being addressed within these studies. While patients as teachers of examination skills may be on a par with their clinical counterparts, the fact remains that the patient as teacher in this circumstance is not necessarily adding anything to the teaching experience that students could not get from anyone else. ${ }^{27}$ Therefore, the main purpose of this initiative could be seen as one of reducing teaching costs, or providing teaching where student access to training is limited while maintaining quality teaching experiences. While this is an acceptable rationale in the face of teacher shortages and financial issues, student attitudes toward these initiatives is less enthusiastic.

The role of patients as teachers of the biopsychosocial model of disease and lived experience of disease is unique to the patient and cannot be replicated by others. Therefore, patients teaching through personal illness narratives are (so far) the best use of patient teaching time.

\section{Limitations of the literature}

We have established that patients as teachers of the biopsychosocial model of disease and their lived experiences are unique and powerful learning experiences for students, and those patients can also benefit from their role as teachers.

However, the majority of studies use student-satisfaction questionnaires or self-reported learning outcomes at the time of the intervention as their evaluation. ${ }^{38}$ Spencer et al noted in their 2011 report for the Health Foundation that while many short-term benefits are quoted in patient-involvement studies, there is a lack of evidence on the long-term impact of these initiatives. ${ }^{20}$ It is not clear if these experiences stay with students in the long-term, influencing the way they think about and practice medicine after graduation and beyond. In addition, the vast majority of studies include patient narratives/ teaching as a one-off event, rather than a longitudinal theme running throughout the curriculum. ${ }^{20}$

\section{Patients as curriculum designers and developers}

The level of patient involvement in curricula design and development is still generally low across the sector. Very few studies identified in Jha et al's 2009 systematic review detail patient involvement in curricula design $n^{39,40}$ or development, ${ }^{41,42}$ and at present there is no research detailing why this remains an underrepresented area.

It is possible to suggest potential reasons for this. In practical terms, facilitating patient involvement in these areas requires careful consideration of where and when (in physical and educational terms) patients can contribute most effectively. Simply inviting patients to sit on school committees does not necessarily translate into patient involvement. Changes to how we discuss education may be required, or patients may need assistance in accessing and understanding the academic world and the language we speak. Realistically, expertise in working with patients within higher education is required to ensure patients' needs are met as well as educational needs. This expertise is not widely available. There are additional issues, 
such as cost of patient involvement (payments, expenses, carers, interpreters, etc) and environmental issues to consider, such as access, length of meetings, and development of an appropriate supportive environment.

Along with these practical considerations are the more challenging philosophical issues: Is there a real desire within the organization to have patients as partners in education? Is the concept of patients as partners valued by those in senior academic roles? Are patients as partners seen as a threat not just within the clinical environment but also by medical educators? A threat to the power and hierarchy within higher-education institutions, or a threat to educator identity? These questions demonstrate the tension between the drive for patient involvement theoretically and its implementation in practice.

\section{Discussion}

When we consider what we want students to gain from their experience of patient involvement in education, it actually goes far beyond the "patient perspective". A student passively listening to a patient talking about how diabetes or osteoarthritis affect their daily lives and their experiences with the NHS and medical professionals can be a powerful learning experience, but what happens next?

What we as medical educators are trying to achieve through this kind of learning is not just the student recognizing that living with diabetes is harder than they imagined; we want them to develop empathy and become "patientcentered" practitioners.

Mead and Bower ${ }^{30}$ identify five key aspects of "patientcenteredness": biopsychosocial perspective, "patient as person", sharing power and responsibility; "therapeutic alliance", and "doctor as person". To become patient-centered, students must actively use what they have learnt from patient narratives to inform how they think about disease and its management (a biopsychosocial model), how they interact with patients in the future (doctor as person), how they involve patients in choices regarding their care and treatment (sharing power and responsibility), how they develop beneficial relationships with patients (therapeutic alliance), and how patients need to be treated as individuals when considering interventions (patient as person). This is a lot to expect from students following one or two interventions.

Effectively, the purpose of patients as teachers using their own illness narratives is to stimulate a change in thinking and attitudes. This is transformative learning, ie, learning that effects change within our frame of reference. ${ }^{43}$ Mezirow explains that frames of reference are the "structure of assumptions through which we understand our experiences". ${ }^{43}$ These are made up of "habits of mind" and "a point of view". Habits of mind are the general ways we think about the world based on our culture, education, etc, and points of view are how we articulate these habits of mind. Points of view change often when we encounter situations that do not unfold as we imagined they would. Habits of mind are much more ingrained. Mezirow asserts that we "transform our frames of reference through critical reflection on the assumptions upon which our [...] habits of mind or points of view are based". ${ }^{43}$ The most common way that this is achieved is through discourse.

To become a patient-centered medical student, students must change their frame of reference from a purely biomedical understanding of medicine to a biopsychosocial understanding. The degree to which that is a change to habit of mind or just a refinement of point of view is different to each individual medical student.

We do not mean to imply by this that all students view medicine only in scientific and diagnostic terms; however, the necessary underpinning knowledge required to practice medicine is vast, and many students lose sight of the humanistic purpose of medicine during their training. ${ }^{44}$

The way in which students often report their reaction to patient narratives using such terms as "powerful, memorable, fantastic, and inspirational"17 illustrates that some students experience change to this frame of reference straight away, but how long does this attitude last: till the next task, exam, or forever? This is where the literature in this field needs further development. In their randomized controlled trial piloting the feasibility and acceptability of patient-led training on patient safety, Jha et $\mathrm{al}^{27}$ attempted to evaluate the impact of patientled patient-safety training on trainee practice 6 weeks after the intervention. While some evidence of impact was found, poor response rates from trainees in the follow-up limited how representative this was.

There is also the issue of students who are not affected by patient narratives, or do not wish to change their frame of reference. How do we know who they are, and does it matter? Follow-up, facilitator-led workshops designed to unpack these patient-narrative experiences and their impact on future practice would help students to turn the learning experience from enjoyable to transformative.

Kumagai $^{45}$ provided a more longitudinal, thoughtful approach to the use and impact of patient narratives in medical education. Kumagai used psychological theory ${ }^{45}$ and patient narratives as transformative learning experiences with the aim of developing empathy, moral development, and patient-centeredness among medical students. 
Through a 2-year longitudinal program of home visits to the same family, students appreciate what it is like to experience illness, and develop an "interpersonal link in affective, cognitive, experiential domains", ${ }^{45}$ which stimulates "other directed role taking" (ie, imagining what that illness and experience might feel like). Sometimes, these interactions result in "emotional or cognitive dissonance", whereby students experience challenges to their frame of reference. The strength of the narrative lies in universally experienced emotional responses triggered in others by the story (loss, anger, jealousy, guilt, and sadness). This trigger builds a bridge between the patient and students' worlds, arousing feelings of empathy and on occasions a desire for social "justice" for the patient. These visits are supported by facilitated discussion sessions and student engagement in reflective practice as individuals and in groups. Patient views of the student are also incorporated into this assessment.

This approach to patient involvement in teaching and assessment demonstrates a much higher level of commitment to patient involvement within the institutional culture than is generally seen in medical education programs. The qualitative evaluations (focus groups and one-to-one interviews with medical students) undertaken presented evidence that this approach not only provides the student with the traditional biomedical knowledge of chronic disease but also additionally delivers "personal, affective, and experiential knowledge acquired through the students' relationships with patients and their families". ${ }^{45}$ While further longitudinal impact studies would strengthen the evidence of this initiative, it is clear that the aim of the patient-involvement program is achieved.

The educational design of this program enhances the impact of patient-led education, due to the longitudinal nature of the initiative and the placement of students with one family for the duration of the program. The facilitated discussions and patient input into assessment demonstrates to students that faculty value patients as educators. The initiative is not tokenistic, as many "one off" patient-narrative events can be perceived. These design features are far more likely to achieve the patient-centered attitudes we are looking to develop among our medical students.

Additionally, the qualitative research methods used to evaluate the program allow a depth-of-impact evaluation from individual students that cannot be explored using satisfaction questionnaires. More qualitative evaluation studies in this vein would help to address some of the current limitations to the literature within the patient-involvement field.
While the content of undergraduate medical education programs is always under time and resource constraints, more embedded patient-led education initiatives, such as Kumagai's, are far more likely to achieve the drive within health care policy and delivery to provide patient-centered care than "wheeling patients in" to university settings.

\section{Future directions}

In this review, we have examined the roles patients currently play in medical education, primarily that of teacher and facilitator of learning experiences. These roles are a start on the journey to patient partnership ${ }^{28}$ and the achievement of true patient-centeredness in medical education. However, this remains a long way off, as it requires a significant paradigm shift on the part of medics, medical educators, institutions, and health care structures. Such initiatives as the Expert Patients Programme and the use of patients as teachers demonstrate the start of a shift; however, until we understand the impact of the individual, community of practice, institution, and governance on these initiatives, we cannot start to reconstruct ingrained cultures and stimulate true change. The theoretical research conducted by Rees et al, ${ }^{17,18}$ Monrouxe et al, ${ }^{19}$ Mead and Bower, ${ }^{30}$ and Kumagai ${ }^{45}$ help us to view and understand the everyday culture of medicine and medical education in a way that can identify how to effect this change. More theoretically based research from a variety of disciplines could help to move the patient-involvement agenda forward, empowering patients and developing the concept of medicine and how it is practiced.

\section{Disclosure}

The authors report no conflicts of interest in this work.

\section{References}

1. Cleland JA, Abe K, Rethans JJ. The use of simulated patients in medical education: AMEE Guide No 42 1. Med Teach. 2009;31(6):477-486.

2. Anderson M, Stillman PL, Wang Y. Growing use of standardized patients in teaching and evaluation in medical education. Teach Learn Med. 1994;6(1):15-22.

3. Maran NJ, Glavin RJ. Low- to high-fidelity simulation - a continuum of medical education? Med Educ. 2003;37 Suppl 1:22-28.

4. Ladyshewsky R. Simulated patients and assessment. Med Teach. 1999;21(3):266-269.

5. Pell G, Fuller R, Homer M, Roberts T. How to measure the quality of the OSCE: a review of metrics - AMEE guide no 49. Med Teach. 2010; 32(10):802-811.

6. Kilminster S, Roberts T, Morris P. Incorporating patients' assessments into objective structured clinical examinations. Educ Health (Abingdon). 2007;20(1):6.

7. Miller GE. The assessment of clinical skills/competence/performance. Acad Med. 1990;65(9 Suppl):S63-S67.

8. Cook DA, Triola MM. Virtual patients: a critical literature review and proposed next steps. Med Educ. 2009;43(4):303-311. 
9. Stevens A, Hernandez J, Johnsen K, et al. The use of virtual patients to teach medical students history taking and communication skills. Am J Surg. 2006;191(6):806-811.

10. Towle A, Bainbridge L, Godolphin W, et al. Active patient involvement in the education of health professionals. Med Educ. 2010;44(1): 64-74.

11. Jha V, Quinton ND, Bekker HL, Roberts TE. Strategies and interventions for the involvement of real patients in medical education: a systematic review. Med Educ. 2009;43(1):10-20.

12. Department of Health. Equity and Excellence: Liberating the NHS. London: Department of Health; 2010.

13. NHS England. Planning and Delivering Service Changes for Patients: A Good Practice Guide for Commissioners on the Development of Proposals for Major Service Changes and Reconfigurations. Leeds: NHS England; 2013.

14. Albanese M, Mejicano G, Gruppen L. Perspective: Competency-based medical education: a defense against the four horsemen of the medical education apocalypse. Acad Med. 2008;83(12):1132-1139.

15. Lave J, Wenger E. Situated learning: Legitimate Peripheral Participation. Cambridge: Cambridge University Press; 1991.

16. Goffman, E. The Presentation of Self in Everyday Life. London: Penguin; 1990.

17. Rees CE, Knight LV, Wilkinson CE. "User involvement is a sine qua non, almost, in medical education": learning with rather than just about health and social care service users. Adv Health Sci Educ Theory Pract. 2007;12(3):359-390.

18. Rees CE, Knight LV, Wilkinson CE. Doctors being up there and we being down here: a metaphorical analysis of talk about student/doctorpatient relationships. Soc Sci Med. 2007;65(4):725-737.

19. Monrouxe LV, Rees CE, Bradley P. The construction of patients' involvement in hospital bedside teaching encounters. Qual Health Res. 2009;19(7):918-930.

20. Spencer J, Godolphin W, Karpenko N, Towle A. Can Patients be Teachers? Involving Patients and Service Users in Healthcare Professionals'Education. London: Health Foundation; 2011.

21. Barrows HS, Abrahamson S. The programmed patient: a technique for appraising student performance in clinical neurology. J Med Educ. 1964;39(8):802-805.

22. Helfer RE, Black MA, Teitelbaum H. A comparison of pediatric interviewing skills using real and simulated mothers. Pediatrics. 1975;55(3): $397-400$.

23. Eagles JM, Calder SA, Nicoll KS, Walker LG. A comparison of real patients, simulated patients and videotaped interview in teaching medical students about alcohol misuse. Med Teach. 2001;23(5): 490-493.

24. Klein S, Tracy D, Kitchener HC, Walker LG. The effects of the participation of patients with cancer in teaching communication skills to medical undergraduates: a randomised study with follow-up after 2 years. Eur J Cancer. 1999;35(10):1448-1456.

25. Jha V, Quinton ND, Bekker HL, Roberts TE. What educators and students really think about using patients as teachers in medical education: a qualitative study. Med Educ. 2009;43(5):449-456.

26. Bell K, Boshuizen H, Scherpbier A, Dornan T. When only the real thing will do: junior medical students' learning from real patients. Med Educ. 2009;43(11):1036-1043.
27. Jha V, Winterbottom A, Symons J, et al. Patient-led training on patient safety: a pilot study to test the feasibility and acceptability of an educational intervention. Med Teach. 2013;35(9):e1464-e1471.

28. Tew J, Gell C, Foster S. Learning from Experience: Involving Service Users and Carers in Mental Health Education and Training. Nottingham: Higher Education Academy/NIMHE West Midlands/Trent Workforce Development Confederation; 2004.

29. Raj N, Badcock LJ, Brown GA, Deighton CM, O’Reilly SC. Undergraduate musculoskeletal examination teaching by trained patient educators - a comparison with doctor-led teaching. Rheumatology. 2006;45(11):1404-1408.

30. Mead N, Bower P. Patient-centredness: a conceptual framework and review of the empirical literature. Soc Sci Med. 2000;51(7): 1087-1110.

31. Daykin N, Rimmer J, Turton P, et al. Enhancing user involvement through interprofessional education in healthcare: the case of cancer services. Learn Health Soc Care. 2002;1(3):122-131.

32. Felton A, Stickley T. Pedagogy, power and service user involvement. J Psychiatr Ment Health Nurs. 2004;11(1):89-98.

33. Szasz TS, Hollender MH. A contribution to the philosophy of medicine: the basic models of the doctor-patient relationship. AMA Arch Intern Med. 1956;97(5):585-592.

34. Chretien KC, Goldman EF, Craven KE, Faselis CJ. A qualitative study of the meaning of physical examination teaching for patients. J Gen Intern Med. 2010;25(8):786-791.

35. Stillman PL, Ruggill JS, Rutala PJ, Sabers DL. Patient instructors as teachers and evaluators. J Med Educ. 1980;55(3):186-193.

36. Stacy R, Spencer J. Patients as teachers: a qualitative study of patients' views on their role in a community-based undergraduate project. Med Educ. 1999;33(9):688-694.

37. Mukohara K, Ban N, Sobue G, Shimada Y, Otani T, Yamada S. Follow the patient: process and outcome evaluation of medical students' educational experiences accompanying outpatients. Med Educ. 2006;40(2): $158-165$.

38. Morgan A, Jones D. Perceptions of service user and carer involvement in healthcare education and impact on students' knowledge and practice: a literature review. Med Teach. 2009;31(2):82-95.

39. Blasco PA, Kohen H, Shapland C. Parents-as-teachers: design and establishment of a training programme for paediatric residents. Med Educ. 1999;33(9):695-701.

40. Smith MD, Henry-Edwards S, Shanahan EM, Ahern MJ. Evaluation of patient partners in the teaching of the musculoskeletal examination. J Rheumatol. 2000;27(6):1533-1537.

41. O'Keefe M, Jones A. Promoting lay participation in medical school curriculum development: lay and faculty perceptions. Med Educ. 2007;41(2):130-137.

42. Chur-Hansen A, Koopowitz L. The patient's voice in a problem-based learning case. Australas Psychiatry. 2004;12(1):31-35.

43. Mezirow J. Transformative learning: theory to practice. New Dir Adult Contin Educ. 1997;1997(74):5-12.

44. Wear D, Nixon LL. Literary inquiry and professional development in medicine: against abstractions. Perspect Biol Med. 2002;45(1): 104-124.

45. Kumagai AK. A conceptual framework for the use of illness narratives in medical education. Acad Med. 2008;83(7):653-658.
Innovation and Entrepreneurship in Health

\section{Publish your work in this journal}

Innovation and Entrepreneurship in Health is an international, peer reviewed, open access journal publishing original research, reports, reviews and commentaries on innovation and entrepreneurship in health. Special focus will be given to the theory, process, and practice of innovation and entrepreneurship by individuals and organizations

\section{Dovepress}

within the health care context globally. The manuscript management system is completely online and includes a very quick and fair peer review system, which is all easy to use. Visit http://www.dovepress.com/ testimonials.php to read real quotes from published authors. 